\title{
SUPPORTING AWARENESS IN CREATIVE GROUP WORK BY EXPOSING DESIGN RATIONALE
}

\author{
Umer Farooq \\ Microsoft Corporation \\ USA
}

\author{
John M. Carroll \\ The Pennsylvania State University \\ USA
}

\begin{abstract}
When creativity is taken as a long-term, complex, and collaborative activity, support for awareness is required for group members to monitor the development of ideas, track how these ideas became narrowed, and understand how alternatives are being implemented and integrated by colleagues. In this paper, we investigate the effects of exposing design rationale to convey awareness, specifically activity awareness, in group creativity. Through evaluating a prototype, we investigate status updates that convey design rationale, and to what consequences, in small groups in fully distributed collaboration. We found that status updates are used for a variety of purposes and that participants' comments on their collaborators' status updates provided feedback. Overall, results suggest that participants' awareness about their collaborators' future plans increased over time. Majority of participants found the status updates useful, particularly those with higher metacognitive knowledge. Based on our results, two design strategies for activity awareness are proposed.
\end{abstract}

Keywords: computer-supported awareness, status updates, metacognition, Facebook, Twitter.

\section{INTRODUCTION}

A fairly typical form of creativity-and perhaps even more important than the isolated lightning bolt - is the relatively long-term and collaborative development of innovative ideas. Researchers have argued in prior work that computer-supported awareness is critical for successful collaborative work, as well as creative work (Farooq, Carroll, \& Ganoe, 2007).

The concept of awareness in computer-supported cooperative work (CSCW) literature has taken many forms. For example, social awareness (Erickson \& Kellogg, 1999) involves knowing who else is present in a shared workspace, while workspace awareness (Gutwin, Greenberg, \& Roseman, 1996) conveys who is doing what in the sense of manipulating shared artifacts. For a detailed review of awareness in CSCW, refer to Schmidt (2002).

(C) 2011 Umer Farooq and John M. Carroll, and the Agora Center, University of Jyväskylä URN:NBN:fi:jyu-2011081711242 
More recently, with the adoption of Web 2.0, new social networking media have emerged that incorporate novel ways of providing awareness of people's activities. For instance, Facebook, an on-line social networking site, allows friends to specify what they are doing through status updates. These status updates are broadcasted to one's friends. Twitter is yet another social networking medium that among many functions serves the purpose of keeping friends, family, and colleagues connected through the exchange of quick, frequent answers to one simple question: What are you doing right now?

The notion of providing status updates for informal, nonwork related purposes, such as in social networking media (e.g., Facebook, Twitter), seems to be anecdotally successful in supporting awareness. For example, Farooq et al. (2007) showed that different types of breakdowns in creativity do occur in distributed collaboration and can be supported by designing appropriate awareness mechanisms around status updates.

We thus are motivated to investigate the feasibility, effectiveness, and consequences of such status updates in the context of creative group work. In this paper, we describe a prototype for updating statuses in collaborative work. We report on a study of small groups using this prototype over an extended period of time to answer the exploratory research question: How can status updates be used in creative group work and with what consequences?

The outline of this paper is as follows. First, we conceptualize status updates as providing activity awareness in collaborative work. Second, we discuss how our contribution is situated in prior work on status updates in CSCW. Then, based on an existing empirical study, we describe the design of a status update prototype that provides activity awareness in collaborative work. Finally, we present an evaluation of the prototype. We conclude by suggesting implications of our results and future work.

\section{EXPOSING DESIGN RATIONALE AS STATUS UPDATES TO SUPPORT ACTIVITY AWARENESS}

We are investigating the provision of status updates to convey activity awareness in creative group work. Activity awareness is cognizance of other people's intentions, plans, priorities, and understandings with respect to an ongoing endeavor, of the criteria they will use to make decisions and evaluate joint outcomes, of the knowledge, skills, tools, and other resources they can contribute to a joint project, of the social networks they participate in, and of how they can engage others in the shared activity (Carroll, Neale, Isenhour, Rosson, \& McCrickard, 2003; Carroll, Rosson, Convertino, \& Ganoe, 2006).

By definition (Carroll et al., 2003; Carroll et al., 2006), activity awareness is more than a matter of registering the current state information. It transcends synchronous awareness of where a collaborator's cursor is pointing, where the collaborator is looking, and so forth. It involves monitoring and integrating many different kinds of information at different levels of analysis, such as events, tasks, goals, social interactions and their meanings, group values and norms, and more to learn about developing circumstances and the initiatives, reactions, as well as sense making of other people with respect to ongoing and anticipated courses of action. It is continually negotiated, constructed, and enacted throughout the course of a collaborative interaction. 
Currently, status updates in social networking media are employed to present general and often lighthearted self-disclosures (e.g., "Joe is glad the semester is over"; "Kathy is craving pizza"). However, one can imagine recruiting the mechanism of status updates for the purpose of supporting activity awareness in collaborative work; thus, a group member might post a status update such as, "Sam is finishing up Section 3 of the heuristic evaluation report." In this application, a status update is specific and activity-oriented. Of course, this single status update is just an excerpt from a presumably more extensive signaling protocol but, even so, it reminds group members of the planning and negotiation that divided up report sections, and of the ensuing steps in the plan. It conveys something about Sam's progress, and about what other group members may need to be doing in order to coordinate with Sam.

In the balance of this paper, we will refer to status updates that are intended to support activity awareness as activity updates. Our perspective on activity updates is that the updates actually constitute design rationale. Each team member keeps his/her partners apprised of that member's activities and reactions through the updates. This puts the partners' actions within the intentional context of what they are trying to do, how they assessed what others are trying to do, and so on. The updates are rationale-in-action, or situated rationale, as opposed to more typical case of rationale created after the fact to reconstruct or explain what happened.

\section{RELATION TO PRIOR LITERATURE}

Researchers in several CSCW studies have investigated the use of status updates in collaborative contexts. For example, Tickertape (Fitzpatrick, Parsowith, Segall, \& Kaplan, 1998) is a lightweight awareness tool to facilitate social interaction between coworkers. The Notification Collage (Greenberg \& Rounding, 2001) is a full-fledged groupware system that incorporates the notion of activity indicators through a variety of media, such as digital photos and video. The Community Bar (McEwan \& Greenberg, 2005) extends the Notification Collage by supporting communities in fostering and maintaining ad hoc interaction.

Although there are some similar attributes between our structured activity updates prototype and other systems, there are also significant differences. Primarily, we are studying awareness of longer term activities as intentionally broadcasted by users in the context of fully distributed work, not social settings. For example, Smale and Greenberg (2005) studied status updates among instant messenger clients. Though their study context was not collaborative work, it is interesting to note that some of their communication categories of status updates are similar to ours. In their categorization scheme, "fun" is a type of status update that overlaps with our findings. Particular to our study context, Smale and Greenberg noted that people use status updates to broadcast information without involving chat conversation. This supports the feasibility of recruiting status updates for distributed collaborative work as a natural extrapolation from their original intended use in social networking media.

In their paper, Zhao and Rosson (2009) presented a complementary contribution to our work. They retrospectively investigated why people microblog and its potential impact on informal communication in work environments. Those authors suggested that microblogging may promote informal communication that complements other forms of interaction (e.g., IM, e-mail, phone, face-to-face). 
Perhaps the work most related to ours is the study by Rittenbruch, Viller, and Mansfield (2007). In presenting their model of intentionally enriched awareness, Rittenbruch et al. reviewed and critiqued prior accounts of awareness as ignoring the ways that actors deliberately present themselves and their activity to collaborators. Rittenbruch et al. located intentionally enriched awareness as lying between mere perception of appearances and events and the public communication and explanation of one's activity.

Rittenbruch et al. (2007) focused on notifications of interest and availability for specific activities, such as playing a computer game or going for a coffee. They developed a tool to configure activity-specific polling and notification, enabling users not only to signal their own availability and interest, but to coordinate carrying out the activity with other likeminded users. This tool was used by users who were colocated. Rittenbruch et al. categorized the status notifications as activity indicators and activity inducements. Activity indicator notifications act as invitations to announce that certain activities are about to commence and that fellow users are invited to jointly participate. Activity inducement notifications are statements to convey that people are already engaged in activities.

\section{DESIGN OF ACTIVITY UPDATES}

The empirical study that we report in this paper addresses our overall research question, which we restate as, how can activity updates be used in creative group work and with what consequences? We evaluated a prototype that supports status updates in the context of collaborative work by conveying activity awareness. The design of our prototype is based on the Farooq et al. (2007) study.

\section{Activity Updates as Structured Templates}

Farooq et al. (2007) conducted an empirical study of small groups collaborating on a long-term, creative task in a distributed setting. The researchers concluded that members in distributed groups lacked activity awareness that would otherwise be critical not just for successful but also creative collaboration. A design implication emerging from this study was the notion of structured activity updates, whereby team members can enter status updates using predefined activity-centric templates that relate to their current work. An example of a structured activity update would be to allow Sam to fill in the blanks in the following activity-centric template: "Sam is planning to ." The activity updates are structured in the sense that each group member is presented with a template to update his/her status.

Whereas social networking sites allow users to enter any type of informational update, our proposed updates are activity-centric, that is, they relate to the collaborators' task at hand. By structuring the updates in predefined activity-centric templates, we believe users will be more inclined to think in terms of what they are doing with respect to their immediate task and less inclined to socially loaf and focus on nonwork-related self-disclosures. This is consistent with Malone, Grant, Lai, Rao, and Rosenblitt's (1986) finding that semistructured messages can simplify designing systems that (a) help people formulate information they wish to communicate, (b) automatically select, classify, and prioritize information people 
receive, (c) automatically respond to certain kinds of information, and (d) suggest actions people may wish to take upon receiving certain other kinds of information.

A structured approach to activity updates has its associated trade-offs. For instance, entering activity updates may be too constraining and become an impediment in the users' workflow and creative process. While acknowledging this trade-off, our rationale for keeping the activity updates structured was specifically to make it convenient for users to quickly select from a predefined list of activity templates, rather than ruminating over the nature of their activities.

\section{Types of Activity Updates}

Given the rationale for making activity updates structured, the next step was to identify the types of activity updates. We followed an empirical approach to achieve this goal. Based on the data collected from Farooq et al.'s (2007) study, we analyzed the chat communication transcripts to identify the different types of activities that collaborators expressed and shared. The primary researcher in this paper read and reread each chat communication transcript to identify recurring themes related to activities that group members were engaged in consistently. These 10 types of activities were then discussed and revalidated with two other researchers engaged in the project. Ten distinct types of activities were identified that would serve as templates for group members to update their status: planning, brainstorming, working, asking, suggesting, summarizing, dividing up work, proofreading, agreeing, disagreeing.

A trade-off to empirically identifying the types of activity updates was that the 10 types conveyed activity self-disclosures at different levels of detail and generality. For instance, working is a relatively broad activity compared to, say, proofreading, which is a very specific activity. We decided not to tweak these empirically based activities (e.g., relabel the activities at a similar level of detail) since, as a secondary research question, it may be interesting to understand how users update activities that vary in granularity. Further, because the 10 activity types emerged from a prior study, we took them as first-order, validated approximations to the kinds of activities group members would be engaged in.

\section{Prototype}

Based on our analysis, we designed and developed a prototype for structured activity updates (Figure 1). The structured activity updates prototype can be divided into three sections.

In Section 1 (Update Your Activity), users can choose from among the 10 activity templates and fill in the blanks to share their activities. The 10 activity types were presented in random order. After a user fills in the blank and presses the Update Activity button, the activity updates are displayed in Section 2 of the prototype. In addition to the team's most recent updates, each user's prior activity update is also displayed. We thought that providing a user's previous activity would be useful in contextualizing the current activity.

Section 3 of the prototype provides a mechanism for users to comment on group members' activity updates. Our design rationale was that activity updates could instigate and provoke users to reflect on and possibly respond to group members' activities. By commenting on others' activities, group members could provide feedback and possibly engage in a discourse. For instance, in Section 3, Patti commented on Michael's activity update, which led Kristin to agree with Patti's comment. 


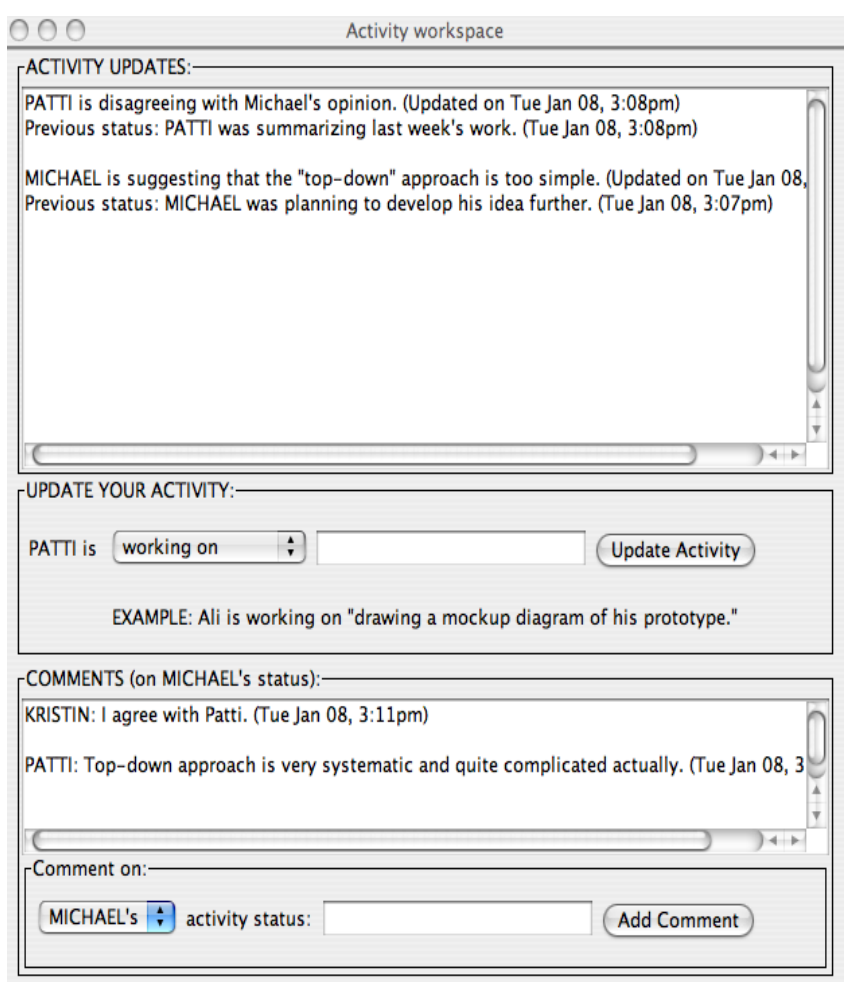

Figure 1. Structured activity updates prototype.

\section{EXPERIMENTAL STUDY}

We conducted an empirical study to investigate the use and consequences of structured activity updates. The context was similar to that of Farooq et al.'s (2007) study, where small groups collaborated on a long-term, creative task in a distributed setting.

\section{Research Questions}

To answer our overall research question, we formulated specific questions in the following two categories: use of structured activity updates (USE) and consequences of structured activity updates (CONSQ). In the former category (USE), we present results that answer the following three questions:

USE1: How frequently were the different types of activity updates used?

USE2: For what purposes were the activity updates used?

USE3: For what purposes were the activity comments used?

USE1 helps to distill any significant differences in the usage among the 10 types of structured activity updates. USE2 and USE3 help in understanding the different ways in which activity updates and comments were used.

In the CONSQ category, we present results that answer the following three questions:

CONSQ1: Did users find the activity updates useful? 
CONSQ2: Did the activity updates increase awareness over time?

CONSQ3: Did metacognition affect users' perception of the usefulness of activity updates?

CONSQ1 directly answers the question of whether or not the activity updates were useful. CONSQ2 is motivated by the fact that we ultimately are interested in whether or not activity updates had a significant effect on users' awareness over time. We expected that activity updates would enhance awareness. CONSQ3 is motivated by a prior study on activity awareness (Convertino, Neale, Hobby, Carroll, \& Rosson, 2004), which provided preliminary evidence that people's metacognitive abilities can affect the extent to which they become aware. Whereas cognition is the act of knowing, metacognition is the ability to reflect upon, understand, and control that knowledge (Schraw \& Dennison, 1994). Metacognition has also been noted as the awareness and control over one's thinking (Brown, Bransford, Ferrara, \& Campione, 1983; Flavell, 1978; Metcalfe \& Shimamura, 1994). We expected that people with lower metacognition would find the activity updates more useful than those with higher metacognition because the tool would compensate for their lower metacognitive abilities.

\section{Participants}

Participants in the study comprised 49 undergraduate students enrolled in an introductory course on HCI at a large university. A total of 13 groups were randomly assigned. Ten groups comprised 4 students each and three groups comprised 3 students each. All groups had no history of working together but were familiar with status updates, particularly with Facebook.

\section{Task Details}

Each group was instructed to write a formal report exploring design enhancements to Angel, which is the university's course management system that all students must use. The report was to cover functional requirements to enhance the design of Angel's user interface, accompanied with scenarios and storyboards that illustrate the functional requirements. This task was modeled on previously documented tasks (Ocker, Hiltz, Turoff, \& Fjermestad, 1996; Olson, Olson, Storrosten, \& Carter, 1993) used in distributed collaboration. The instructions emphasized that group members were allowed only to collaborate virtually using the designated shared workspace.

\section{Tools}

Group members worked on the collaborative task in a shared workspace called BRIDGE (Ganoe et al., 2003). BRIDGE supports both synchronous and asynchronous collaboration. The BRIDGE Java-based client (Figure 2) supports shared editing of documents through replicated objects. Replicated objects are objects that are retrieved by multiple collaborating sessions and whose state is kept synchronized on all clients and the server when any replica is changed. Examples of collaborative tools that were provided in BRIDGE included a persistent chat tool, wiki-based text documents, and drawing editor. The structured activity updates prototype was also provided. 


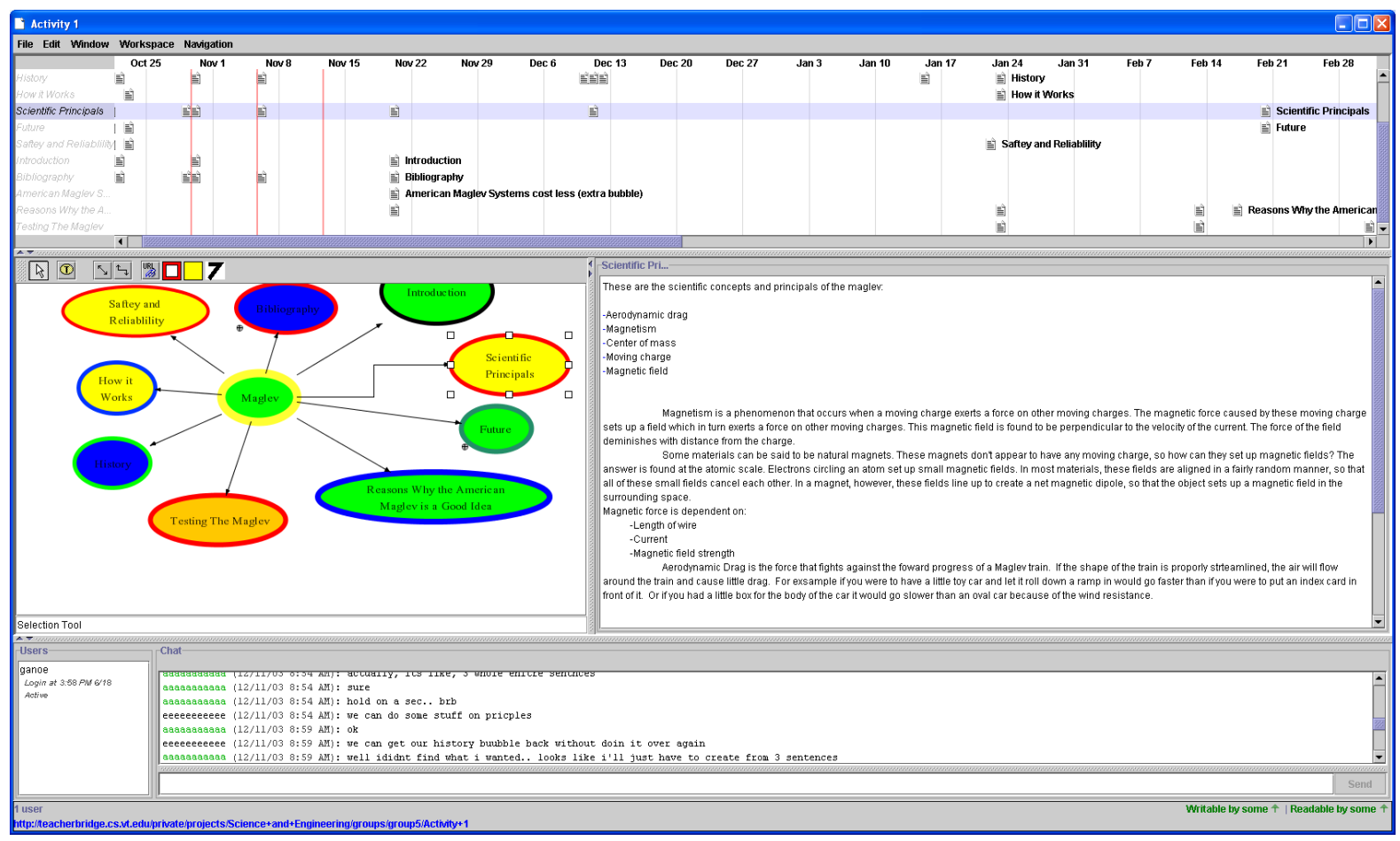

Figure 2. BRIDGE: Basic Resources for Integrated Distributed Group Environments.

\section{Procedure}

All students consented to participate in the experiment. Prior to the experiment, all participants were trained to use BRIDGE. Two in-class sessions, approximately 20 minutes each, were used for training. Training included using the structured activity updates.

The duration of the experiment was two and a half weeks. Participants collaborated synchronously and asynchronously. Five in-class sessions were used for synchronous collaboration, each lasting approximately 20 minutes. The participants also collaborated on-line in out-of-class sessions between each of the in-class synchronous sessions. Participants worked in a distributed fashion; the instructor ensured this for the in-class sessions. For out-of-class sessions, we analyzed the chat transcripts for any indicators of face-to-face collaboration (e.g., if one member referred to a face-to-face meeting); we did not find any such indicators.

Because we were interested in studying the use and consequences of the structured activity updates prototype, participants were reminded to update their activity status. Participants were not compensated for or graded on using the structured activity updates prototype.

Following is a typical scenario of interaction. During an in-class session, "Ben" communicates with his two group members about what Angel's user interface lacks by using the chat tool. While chatting, Ben uses the structured activity updates prototype to change his status to "Ben is brainstorming how Angel can be enhanced" to make his group members cognizant of what he is doing. Ben records a few user interface enhancements in a wiki-based text document. Logging into BRIDGE at night during an out-of-class session, Ben changes his status to "Ben is asking his group members to read his enhancements and provide feedback." In 
the next in-class session, Ben notices that both his group members have commented on his status and have improved upon his ideas.

\section{Data Collection}

When the participants consented at the start of the experiment, we collected background data, such as demographic information. For metacognitive abilities, a previously validated 52 -item scale (Kumar, 1998) was used.

During the experiment, all interactions in BRIDGE were logged onto the server. For example, communication messages in the chat log and changes to shared data were recorded with time stamps. All activity updates and comments on activity updates were recorded over time.

After each of the five synchronous in-class sessions, participants answered the following statements on a Likert scale of 1 (strongly disagree) to 9 (strongly agree) related to their level of awareness (LA). These assessments have been previously validated by Convertino et al. (2004):

LA1: I know what my group members have worked on so far.

LA2: I know what my group members will work on next time.

LA3: I could tell what my group members were working on while we were collaborating synchronously.

LA4: I could tell what my group members were working on while we were collaborating asynchronously.

LA5: I found it difficult to tell what work my group members had done after being absent from my team workspace for at least a day.

LA4 and LA5 were not administered following the first synchronous in-class session since the groups had no history of working together on the task. LA2 was not administered after the fifth synchronous in-class session since it was the final group interaction.

After the experiment, participants were asked the following question in relation to the usefulness of the structured activity updates (SAU) prototype: Was the structured activity updates useful? If yes, how was it useful; provide an example.

\section{RESULTS}

Below, we present our results in relation to the six specific research questions (USE and CONSQ) we posed earlier. Each result is followed by a brief discussion.

\section{USE1: How Frequently Were the Different Types of Activity Updates Used?}

For each of the 49 participants, we counted the frequency of using each of the 10 activity updates. We also categorized the frequency by synchronous (in-class sessions) and asynchronous (out-of-class sessions) modes of communication. Activity updates were used a total of 511 times, with nearly half split across synchronous (252) and asynchronous (259) updates. These frequencies are summarized in Table 1. 
Table 1. Frequency of Activity Updates.

\begin{tabular}{lccc}
\hline \multicolumn{1}{c}{ Activity type } & Total & Sync & Async \\
\hline Working & 170 & 67 & 103 \\
Planning & 99 & 54 & 45 \\
Brainstorming & 76 & 62 & 14 \\
Proofreading & 62 & 9 & 53 \\
Agreeing & 39 & 26 & 13 \\
Suggesting & 23 & 15 & 8 \\
Asking & 20 & 7 & 13 \\
Summarizing & 10 & 4 & 6 \\
Disagreeing & 6 & 4 & 2 \\
Dividing up work & 6 & 4 & 2 \\
\hline
\end{tabular}

We ran a repeated-measures ANOVA to compute any statistically significant differences between the frequencies of the different types of activity updates. The analysis for unequal variances revealed that there were significant differences in the frequencies between the 10 types of activities, $F(1,48)=43.071, p<0.001$, though this was a moderate effect size (etasquared $=0.473)$. Pairwise comparisons for the top four activities revealed that the mean frequency of Working was significantly higher than all other types of activity updates; Planning was significantly different from all except Brainstorming; Brainstorming was significantly different than all except Planning and Proofreading; and Proofreading was significantly different than all except Brainstorming. A similar analysis could not be run against synchronous and asynchronous modes of communication due to sparse data. From Table 1, however, it is apparent that in the synchronous mode of communication, the three most frequently used activities in rank order were Working, Brainstorming, and Planning; in the asynchronous mode of communication, the three most frequently used activities were Working, Proofreading, and Planning.

Discussion. Working was the most frequently used activity update, for which there could be several reasons. Because the structured activity updates prototype was meant to provide activity awareness in the context of work, it seems natural that collaborators would be inclined to select the Working activity to make others aware of their work-related tasks. It is also possible that Working was the type of activity that collaborators most frequently engaged in. The most plausible explanation is that Working is a general adjective that seems to encompass some if not all of the other types of activities, which are more specific in their description. It seems to be the case that semantic granularity plays a vital role in selecting activity updates, a point that we return to toward the end of the paper.

There was also a disparity in frequencies between the synchronous and asynchronous modes of communication. Two activities particularly stand out. Brainstorming was skewed toward the synchronous mode of communication while Proofreading was skewed toward the asynchronous mode of communication. This is not a surprise. Brainstorming is typically a synchronous activity and proofreading is typically an asynchronous activity. 


\section{USE2: For What Purposes Were the Activity Updates Used?}

Based on the postexperiment questions (SAU1 and SAU2), we analyzed the responses using qualitative analysis. All 49 participants responded to both questions. We coded the 98 responses with the goal of identifying the different purposes that the activity updates were used for.

A total of 37 distinct phrases were identified as specifying a purpose and thus were open coded (Strauss \& Corbin, 1998). A total of nine codes emerged from the data that represent different purposes for how the activity updates were used. These purposes are listed in Table 2 with a coded phrase (user quote) that illustrates the purpose.

Discussion. The qualitative analysis reveals that activity updates were used in varying ways. Broadcasting one's activity and staying up to date of what one's group members are doing were expected ways of how activity updates would be used. This is similar to how status updates are used in social networking media. However, activity updates also seemed to be used for coordinating work and task dependencies, understanding group progress cumulatively over time, and tracking group member contributions.

\section{USE3: For What Purposes Were the Activity Comments Used?}

The actual comments that users expressed in the structured activity updates prototype were analyzed using qualitative analysis. We coded all of the comments with the goal of identifying the different purposes that they expressed.

We open coded the comments at the group level of analysis for each of the 13 groups so as to understand any dependencies among group members that the comments were referring to.

Table 2. Different Ways That Activity Updates Were Used.

\begin{tabular}{|c|c|c|}
\hline $\begin{array}{l}\text { Purpose of } \\
\text { activity }\end{array}$ & $\begin{array}{c}\text { No. of } \\
\text { phrases }\end{array}$ & Data example \\
\hline $\begin{array}{l}\text { Group member } \\
\text { update }\end{array}$ & 13 & "Gave a little info on what each member was doing." \\
\hline $\begin{array}{l}\text { Status } \\
\text { broadcast }\end{array}$ & 8 & $\begin{array}{l}\text { "To show my team members what part of the project I was currently } \\
\text { working on." }\end{array}$ \\
\hline $\begin{array}{l}\text { Asynchronous } \\
\text { communication }\end{array}$ & 4 & $\begin{array}{l}\text { "Allowed me to see what each member of my group was working on } \\
\text { while I was not logged on." }\end{array}$ \\
\hline $\begin{array}{l}\text { Group } \\
\text { progress }\end{array}$ & 4 & "Informed me about my group's progress." \\
\hline $\begin{array}{l}\text { Group member } \\
\text { login }\end{array}$ & 2 & "I could see who logged in last." \\
\hline $\begin{array}{l}\text { Synchronous } \\
\text { communication }\end{array}$ & 2 & $\begin{array}{l}\text { "When different tasks were going on simultaneously, it was helpful to } \\
\text { know who was doing what." }\end{array}$ \\
\hline $\begin{array}{l}\text { Task } \\
\text { coordination }\end{array}$ & 2 & $\begin{array}{l}\text { "I logged on to finish scenario } 1 \text {, and saw what other work was } \\
\text { necessary." }\end{array}$ \\
\hline $\begin{array}{l}\text { Member login } \\
\text { frequency }\end{array}$ & 1 & $\begin{array}{l}\text { "Showed how often group members were logging in and making } \\
\text { additions." }\end{array}$ \\
\hline Timeline & 1 & "It was helpful as a timeline." \\
\hline
\end{tabular}


A total of eight codes emerged from the data that represent different purposes for how the comments were used. These purposes are listed in Table 3, accompanied with the following information: the number of groups (No. of groups) that incorporated the particular purpose and the number of phrases (No. of phrases) that were coded.

Feedback was the most frequent purpose for a comment where participants provided feedback to group members, such as, "Nice job of organizing the list." The comments were also heavily used by all groups to coordinate work (e.g., "We will review each other's sections on Wed."), although we distinguish this from Elaboration, which refers to an explication or enhancement of an idea (e.g., "We should come up with a few more asynchronous ideas!").

Twelve of the 13 groups moderately used the comments to encourage and support each other (e.g., "Keep up the good work"). In nine groups, we noticed that the comments represented a communication discourse similar to a conversation that could have occurred using the chat tool. For example, Greg commented on Katie's status: "Can you think of any more functional requirements?"; a few hours later, Ed also commented on Katie's status by following up on Greg's message: 'I'm also trying to figure out some more functional requirements."

A small number of comments were used to remind group members of deadlines or responsibilities (e.g., "Make sure to add your pros and cons"). A small number of comments were also used for nonwork or "fun" purposes (e.g., "I also like surfing") and to reflect on social dynamics during the metamorphosis stage of concluding group work (e.g., "I have enjoyed working with you and the other group members on this project").

Discussion. The qualitative analysis reveals that comments were used in varying ways to support unplanned, brief, and ad hoc communication. The comment feature in the structured activity updates prototype was used not only for the obvious purpose of providing feedback to group members but also served as a secondary mechanism or communication backchannel (Ackerman, 2000) to allow group members to coordinate work, encourage and remind each other, and occasionally discuss nonwork topics. Such casual interactions keep individuals informed about each other in social and professional contexts, reinforce social bonds, and make the transition to tightly coupled collaboration easier (Whittaker, Frolich, \& Daly-Jones, 1994).

Table 3. Different Ways That Activity Comments Were Used.

\begin{tabular}{ccc}
\hline Purpose of comment & $\begin{array}{c}\text { No. of } \\
\text { groups }\end{array}$ & $\begin{array}{c}\text { No. of } \\
\text { phrases }\end{array}$ \\
\hline Feedback & 13 & 292 \\
Coordination & 13 & 55 \\
Encouragement & 12 & 41 \\
Elaboration & 11 & 28 \\
Discourse & 9 & 21 \\
Reminder & 7 & 16 \\
Fun & 5 & 19 \\
Metamorphosis & 2 & 5 \\
\hline
\end{tabular}




\section{CONSQ1: Did Users Find the Activity Updates Useful?}

In the postexperiment question (SAU1), we asked participants whether or not they found the structured activity updates prototype useful. Of the 49 participants, $22(45 \%)$ found the prototype useful, 9 (18\%) found it somewhat useful, and 18 (37\%) did not find the prototype useful.

Some of the participants expanded on why the structured activity updates prototype was not useful. They expressed that the chat communication tool in BRIDGE served the purpose that was intended by the structured activity updates prototype. Among such responses were the following typical user quotes: "We communicated what we were doing through the chat function"; "This could have automatically been extracted from a chat and displayed"; "I could just go into the chat logs and find out the specific task of each person."

Discussion. There were mixed feelings toward the usefulness of the structured activity updates prototype. Our prior research question (USE2) provides strong evidence that participants used the prototype in varying ways. Directly asking participants about the usefulness of the prototype did not solicit an overwhelming positive response. This may be attributed to the fact that the structured activity updates prototype was not integrated with the chat communication tool. Hence, the design required collaborators to expend extra effort to switch between the structured activity updates prototype and the chat communication tool in order to make effective use of both. Many participants who did not find the prototype useful acknowledged this design shortcoming.

\section{CONSQ2: Did the Activity Updates Increase Awareness Over Time?}

We ran a repeated-measures ANOVA to compute any statistically significant differences on each of the five awareness questions (LA1-LA5) over time. There was increased awareness over time for LA2 over the final four in-class sessions: $F(1,44)=13.71, p<.001$ (eta-squared $=0.238$ ). There were no significant differences in the mean rating for LA1, LA3, LA4, or LA5 over time.

Discussion. The result for LA2 implies that the structured activity updates informed group members what their collaborators will work on in the future. This seems to make sense since, in highly interdependent and collaborative tasks, group members require a greater amount of planning to understand what everyone is going to do. LA1, LA3, LA4, and LA5 dealt with understanding what group members already did. The persistent BRIDGE workspace seemed to have provided enough awareness of such an understanding. However, the workspace cannot provide an understanding of what collaborators are going to do next unless such an understanding is externalized by the group members themselves. Such was the case as group members used the structured activity updates to make their collaborators cognizant of what they are going to do.

\section{CONSQ3: Did Metacognition Affect Users' Perception of the Usefulness of Activity Updates?}

We conducted a reliability analysis for the 49 responses to the 52-item metacognition questionnaire. The analysis revealed a high value of reliability (Cronbach's alpha $=0.984$ ). We then separated the 49 participants into two categories based on their responses of whether 
they found the structured activity updates prototype useful or not. Thus, we had 31 participants in the "useful" category and 18 participants in the "not useful" category.

We ran a two-sample $t$-test between the two categories of usefulness (useful, not useful) with metacognition as the dependent variable. The analysis for equal variances revealed no statistically significant difference: $t(47)=-1.53, p=0.133$.

The 52 items in the metacognition questionnaire were then loaded on two factors based on Kumar (1998): metacognitive regulation and metacognitive knowledge. Metacognitive regulation denotes planning, information management, monitoring, debugging, and evaluation. Metacognitive knowledge denotes declarative, procedural, and conditional knowledge. The above two-sample $t$-test for metacognitive regulation as the dependent variable revealed no statistically significant difference: $t(47)=-0.875, p=0.386$. The same two-sample t-test for metacognitive knowledge as the dependent variable revealed a statistically significant difference: $t(47)=-2.137$, $p<0.05$. The mean metacognitive knowledge rating for participants who found the structured activity updates prototype useful $(M=58.76)$ was significantly higher than the participants who did not find the prototype useful $(M=53.92)$.

Discussion. The result that participants with higher metacognitive knowledge found the structured activity updates prototype more useful than those with lower metacognitive knowledge seems counterintuitive. One would expect that the prototype would compensate for lower levels of metacognition and thus be more useful to such participants.

According to Flavell (1979), metacognitive knowledge refers to acquired knowledge about cognitive processes, knowledge that can be used to control cognitive processes, as well as knowledge of one's own learning processes. For example, a person may be aware that study session efforts will be more productive in the early morning when everyone is asleep rather than evening when there are many distractions. In this sense, participants with higher levels of metacognitive knowledge can be opportunistic in identifying and leveraging strategies that can help them monitor their progress. In such cases, participants are using their conditional knowledge about when and where it is appropriate to use strategies or, in our case, awareness tools, to enhance their collaborative learning experience. Such an explanation would lend support for our result.

On the other hand, metacognitive regulation implies checking the outcomes of incorporating strategies and ensuring that cognitive goals have been met. In this sense, our prototype did not explicitly support a cognitive goal or outcome but rather the process of reaching a particular goal or outcome. The result that there was no significant difference in the levels of metacognitive regulation would be corroborated by such an explanation.

\section{IMPLICATIONS FOR ACTIVITY AWARENESS}

In this paper, we started by posing an exploratory research question: How can activity updates be used in collaborative work and with what consequences? Our study suggests that activity updates - a way of providing activity awareness - can be used for several purposes, increasing awareness of what collaborators will do next over time. Further, activity comments seemed to provide an effective communication backchannel to support ephemeral and infrequent collaboration that is contextually dependent on the activity updates. The results also suggest that users with higher levels of metacognitive knowledge perceive activity updates as more useful 
than users with lower levels of metacognitive knowledge. In this section, we discuss implications of our results by reflecting on two design strategies for providing activity awareness.

\section{Activity Awareness Can Support Lightweight, Informal Self-disclosures Through Communication Backchannels}

Though the participants were provided with a formal, primary communication channel (chat), the majority of them found activity updates and comments useful during their collaboration. We argue that communication backchannels that allow the expression of lightweight, informal self-disclosures in work contexts can be an effective way to increase activity awareness, particularly to apprise each other of their future cognition.

From previous work in CSCW in work contexts, we know that it is important to provide a communication backchannel. Our study characterizes one type of communication that this backchannel should support, namely, lighthearted and casual meta-activity information. Typical communication backchannels usually demand social responsiveness. For instance, during video conferencing, chat may be used as a backchannel to complement verbal communication. In such a scenario, group members are expected to oblige chat entries and respond in kind. On the other hand, activity updates are an example of an informal communication mechanism, where group members are merely expressing their thoughts on a task that may or may not warrant further explication or response. In this way, activity updates are a lightweight mechanism to express informal activity-related information that has a formal bearing on the group's primary task. Our results suggest that activity updates did indeed provide activity awareness through lightweight, informal self-disclosures, as they were primarily used to update group members of one's progress. Further, comments provided an additional layer of backchannel communication to express feedback on other's work progress over time.

Our design strategy - the provision of activity awareness through lightweight, informal self-disclosures to enrich formal communication mechanisms such as chat-strengthens prior results. In Rittenbruch et al.'s (2007) study, it was discovered that notification and communication are interleaved processes. Our study extrapolates Rittenbruch et al.'s result by implying that activity updates are appropriate to express through notification mechanisms.

The different purposes for which the activity updates were used in our study are much broader and deeper than the two general categorizations of activity indicators or activity notifications as identified by Rittenbruch et al. (2007). For example, we identified nine different ways of how activity updates were used. Because our prototype incorporated the notion of structured activity updates using predefined templates, we were able to explore details of how users coordinated specific types of activities. Further, the activity comments feature was encouraging to the effect of acting as a communication backchannel in distributed work contexts. Our study also raises deeper, theoretical issues of how awareness is related to people's metacognitive abilities, a connection that has not been studied in the above-mentioned literature.

\section{Activity Awareness Can Engage Users at a Metacognitive Level}

The dominant approach in CSCW has been to conceptualize awareness mechanisms as engaging users at a cognitive level by making them aware of system-based, event-driven information. For example, knowing where a collaborator's mouse is pointing can indeed 
facilitate the immediate coordination of manipulating shared artifacts. Such awareness mechanisms rely on detecting and providing short-term informational states for low-level tasks and subtasks that facilitates users' immediate cognitive goals. Although the provision of such system-based, event-driven information is critical, it is limited to the extent of what people need to, and can be, aware of. Indeed, awareness mechanisms cannot detect and convey people's intentions. In his critique of awareness being construed as a passive process (Dourish, 1997), Schmidt (2002) said that passive awareness is restrictive and it prevents users from engaging in practices to align and integrate their distributed but interdependent activities. Rittenbruch et al. (2007) capitalized on this notion to argue for a more intentionally enriched awareness mechanism where users can explicitly characterize and share their own activities.

In this sense, we argue that activity awareness mechanisms should engage users metacognitively. Activity awareness mechanisms should seek to help users regulate their cognition and think explicitly about their design rationale with respect to learning and work goals. By explicitly characterizing and sharing their activity intentions and rationale, users can engage in and think about deeper work-related interactions such as coordinating responsibilities, managing dependencies, resolving conflicts, and so forth. This allows collaborators to be cognizant of each other at the level of activities, a higher-order function of shorter and immediate tasks and subtasks that are merely system-based, event-driven nuggets of information. This design strategy is consistent with our result that, in general, metacognition plays a role in determining the usefulness of awareness mechanisms. Specifically, people with higher metacognitive knowledge seem to be more strongly oriented toward and are interested in being aware of their collaborators' activity. It follows from this result that capitalizing on and effectively using activity awareness mechanisms may also be a metacognitive strategy. This suggests that, in general, awareness mechanisms should not be limited just to system-based, event-driven information of what is currently going on in a shared workspace, but also that awareness should be about providing cognizance of activities that need to be internalized and monitored over time such that users are prompted to strategically regulate their cognition and manage their knowledge in order to better achieve their overall goals collaboratively.

\section{DISCUSSION AND FUTURE WORK}

As discussed in our results, it is likely that the semantic granularity of the activity types matters. The variability in use of the 10 activity types provides some evidence of this. Many issues are raised due to our design choice. For example, did all participants take the 10 activity types to mean the same thing? How does the frequency of engaging in a specific activity affect the selection of different activity types? Perhaps most importantly, can circumstances be identified where more general activity types are preferred over more specific activity types, and vice versa? Further studies are required to address these issues comprehensively.

Another important question raised by our study is the design choice of structuring the activity updates. Though participants were not asked to comment on this issue, it seems important to reflect on the level of structure imposed by the predefined activity templates. A system that resembles our design choice of structuring the activity updates is the Coordinator (Winograd \& Flores, 1986), a large-scale electronic communication system that enlists participants in a coding procedure by using predefined speech acts aimed at making implicit 
intent explicit. The premise of this procedure was that explicitly identified speech acts are clear, unambiguous, and preferred, because people tend to be vague regarding their own intent and that of others (Suchman, 1994). Though the Coordinator was largely cited as a failure, it remains to be empirically determined if structured activity updates are preferred over unstructured activity updates and under what circumstances.

Another interesting area to explore is the relation between activity updates and the design artifact and process. Our study's scope did not include whether or not activity updates helped interpret the design rationale that shaped the artifact. Essentially, such an exploration could help answer whether activity updates change the design process or quality of the outcome. More interestingly, the relation between metacognition and design rationale could be understood. If one adopts the perspective that design rationale is essentially metacognition about design, subsequent studies can be designed to assess the effects of metacognitive processes on design outcomes.

Our result that activity updates were used in a variety of ways is encouraging in further exploring the generative nature of the predefined activity types. We provided 10 activity types, or speech acts (as in the Coordinator). Investigating a broader range of speech acts that continually emerge from the ground up is a research issue worthy of exploration. Some participants who did not find the activity updates useful strongly suggested that these be identified from their chat entries. For example, collaborative filtering mechanisms can be used to extract activity updates automatically that can then be fine-tuned by users. Unlike our design choice, if the activity types are not limited and are allowed to grow over time, these activity types can be suggested to users by recommender systems. Our result that some activity types are used differently during synchronous versus asynchronous communication can be extrapolated to recommend certain activity types, depending on the type of communication users are engaged in. Other attributes in addition to communication type, such as duration of a work session (e.g., short spurt vs. extended period) or the stage at which a group is operating (e.g., divergent vs. convergent thinking), can be also mined to recommend appropriate activity updates.

One limitation of our study was that the participants were undergraduate students, although such a shortcoming is to be expected in controlled settings that pool a large number of experimental subjects. We plan to conduct a field study where participants of a distributed research group are collaborating on a project over several weeks. Such a study would allow us to investigate our results further in a naturalistic setting.

Our general empirical finding that metacognitive abilities affect perceived usefulness of awareness mechanisms should be investigated further. Our finding is based on one study and is limited to one factor of metacognition (metacognitive knowledge). We plan to study how and when metacognition, and both factors of metacognitive regulation and metacognitive knowledge, have an effect on awareness and whether this effect can be controlled. We encourage designers and researchers interested in supporting awareness to incorporate metacognition as a variable in their user studies.

\section{REFERENCES}

Ackerman, M. S. (2000). The intellectual challenge of CSCW: The gap between social requirements and technical feasibility. Human-Computer Interaction, 15, 179-203. 
Brown, A. L., Bransford, J. D., Ferrara, R. A., \& Campione, J. C. (1983). Learning, remembering, and understanding. In. J. H. Flavell \& E. M. Markman (Eds.), Handbook of child psychology: Vol. 3. Cognitive development (pp. 77-166). Somerset, NJ, USA: John Wiley and Sons.

Carroll, J. M., Neale, D. C., Isenhour, P. L., Rosson, M. B., \& McCrickard, D. S. (2003). Notification and awareness: Synchronizing task-oriented collaborative activity. International Journal of Human-Computer Systems, 58, 605-632.

Carroll, J. M., Rosson, M. B., Convertino, G., \& Ganoe, C. H. (2006). Awareness and teamwork in computersupported collaborations. Interacting with Computers, 18, 21-46.

Convertino, G., Neale, D. C., Hobby, L., Carroll, J. M., \& Rosson, M. B. (2004). A laboratory method for studying activity awareness. In Proceedings of NordiCHI (pp. 313-322). New York: ACM Press.

Dourish, P. (1997, March). Extending awareness beyond synchronous collaboration. Paper presented at the CHI'97 Workshop on Awareness in Collaborative Systems, Atlanta, Georgia, USA. Retrieved July 25, 2011, from http://www.dourish.com/publications/chi97-awareness.html

Erickson, T., \& Kellogg, W. (1999). Social translucence: An approach to designing systems that support social processes. ACM Transactions on Computer-Human Interaction, 7, 59-83.

Farooq, U., Carroll, J. M., \& Ganoe, C. H. (2007). Supporting creativity with awareness in distributed collaboration. In Proceedings of the International SIGGROUP Conference on Supporting Group Work (pp. 31-40). New York: ACM Press.

Fitzpatrick, G., Parsowith, S., Segall, B., \& Kaplan, S. (1998). Tickertape: Awareness in a single line. In Proceedings of CHI (pp. 281-282). New York: ACM Press.

Flavell, J. H. (1978). Metacognitive development. In J. M. Scandura \& C. J. Brainard (Eds.), Structural process theories of complex human behavior (pp. 213-245). Alphen a. d. Rijn, the Netherlands: Sijthoff and Noordhoff.

Flavell, J. H. (1979). Metacognition and cognitive monitoring: A new area of cognitive-developmental inquiry. American Psychologist, 34, 906-911.

Ganoe, C. H., Somervell, J. P., Neale, D. C., Isenhour, P. L., Carroll, J. M., Rosson, M. B., \& McCrickard, D. S. (2003). Classroom BRIDGE: Using collaborative public and desktop timelines to support activity awareness. In Proceedings of UIST (pp. 21-30). New York: ACM Press.

Greenberg, S. \& Rounding, M. (2001). The notification collage: Posting information to public and personal displays. In Proceedings of CHI (pp. 514-521). New York: ACM Press.

Gutwin, C., Greenberg, S., \& Roseman, M. (1996). Workspace awareness support with radar views. In Proceedings of CHI (pp. 210-211). New York: ACM Press.

Kumar, A. E. (1998). The influence of metacognition on managerial hiring decision making: Implications for management development. Unpublished doctoral dissertation, Virginia Polytechnic Institute and State University, USA. Retrieved August 15, 2011, from http://scholar.lib.vt.edu/theses/available/etd-62698-122255/

Malone, T. W., Grant, K. R., Lai, K.-Y., Rao, R., \& Rosenblitt, D. (1986). Semi-structured messages are surprisingly useful for computer-supported coordination. In Proceedings of CSCW (pp. 102-114). New York: ACM Press.

McEwan, G., \& Greenberg, S. (2005). Supporting social worlds with the community bar. In Proceedings of the International SIGGROUP Conference on Supporting Group Work (pp. 21-31). New York: ACM Press.

Metcalfe, J., \& Shimamura, A. P. (Eds.). (1994). Metacognition. Cambridge, MA, USA: The MIT Press.

Ocker, R., Hiltz, S. R., Turoff, M., \& Fjermestad, J. (1996). The effects of distributed group support and process structuring on software requirements development teams: Results on creativity and quality. Journal of Management Information Systems, 12(3), 127-153.

Olson, J. S., Olson, G. M., Storrosten, M., \& Carter, M. (1993). Groupwork close up: A comparison of the group design process with and without a simple group editor. ACM Transactions on Office Information Systems, 11, 321-348.

Rittenbruch, M., Viller, S., \& Mansfield, T. (2007). Announcing activity: Design and evaluation of an intentionally enriched awareness service. Human-Computer Interaction, 22, 137-171.

Schmidt, K. (2002). The problem with "awareness." Computer Supported Cooperative Work, 11, $285-298$.

Schraw, D., \& Dennison, R. S. (1994). Assessing metacognitive awareness. Contemporary Educational Psychology, 19, 460-475.

Smale, S., \& Greenberg, S. (2005). Broadcasting information via display names in instant messaging. In Proceedings of the International SIGGROUP Conference on Supporting Group Work (pp. 89-98). New York: ACM Press. 
Strauss, A., \& Corbin, J. (1998). Basics of qualitative research: Techniques and procedures for developing grounded theory. Thousand Oaks, CA, USA: Sage.

Suchman, L. (1994). Do categories have politics? The language/action perspective reconsidered. Computer Supported Cooperative Work, 2, 177-190.

Whittaker, S., Frolich, D., \& Daly-Jones, O. (1994). Informal workplace communication: What is it like and how might we support it? In Proceedings of CSCW (pp. 131-138). New York: ACM Press.

Winograd, T., \& Flores, F. (1986). Understanding computers and cognition: A new foundation for design. Norwood, NJ, USA: Ablex Publishing Corporation.

Zhao, D., \& Rosson, M. B. (2009). How and why people Twitter: The role that micro-blogging plays in informal communication at work. In Proceedings of the International SIGGROUP Conference on Supporting Group Work (pp. 243-252). New York: ACM Press.

\section{Authors' Note}

This research was supported by the US National Science Foundation under award IIS-0749172.

All correspondence should be addressed to

Umer Farooq

Microsoft Corporation

One Microsoft Way

Redmond, WA 98052 USA

umfarooq@microsoft.com

Human Technology: An Interdisciplinary Journal on Humans in ICT Environments

ISSN 1795-6889

www.humantechnology.jyu.fi 